J. Phys. IV France 138 (2006) 285-295

(C) EDP Sciences, Les Ulis

DOI: $10.1051 /$ jp4:2006138033

\title{
Systèmes d'imagerie à haute résolution dans le domaine des Rayons X pour le Laser Mégajoule
}

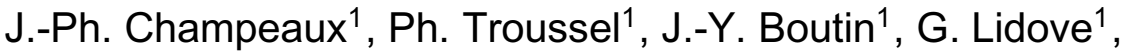 \\ R. Marmoret ${ }^{1}$, G. Soullié ${ }^{1}$ et R. Rosch ${ }^{1}$ \\ ${ }^{1}$ Commissariat à l'Énergie Atomique (CEA) - Direction des Applications Militaires \\ Ile de France (DIF), BP. 12, 91690 Bruyères-Le-Châtel, France
}

\begin{abstract}
Résumé. Le principe de la fusion par confinement inertiel consiste à faire imploser une cible sphérique de petite taille, millimétrique, remplie d'un mélange de deutérium et de tritium sous l'action des 240 faisceaux lasers impulsionnels de très grande puissance du LMJ. Les études de cette physique d'implosion (mise en vitesse, symétrie d'implosion, instabilités hydrodynamiques) et de l'allumage (traceurs, point chaud) font appel aux techniques de l'imagerie et de la radiographie à haute résolution spatiale $(\mu \mathrm{m})$ et temporelle (ps) avec sélection spectrale dans le domaine d'énergie de photons comprise entre $100 \mathrm{eV}$ et $20 \mathrm{keV}$. Plusieurs

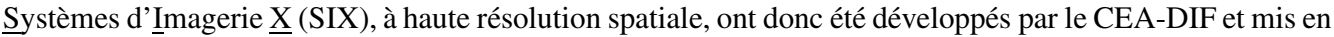
œuvre sur la Ligne d'Intégration Laser (LIL) implantée au CEA-CESTA. Dans cet article nous présentons les principaux SIX, à base de microscopes à miroirs. Un exemple de microscope prototype nommé PIXEL (Plasma Imageur $\underline{X}$ pour les Expériences Lasers) développé pour le LMJ est présenté. Nous portons l'accent sur les problèmes technologiques liés à l'environnement LMJ.
\end{abstract}

\section{INTRODUCTION}

Le principe des expériences de Fusion par Confinement Inertiel (FCI) consiste à réaliser l'implosion d'une petite bille de $1 \mathrm{~mm}$ de diamètre contenant un mélange de gaz légers, par exemple un mélange Deutérium - Tritium (D-T), en vue d'obtenir des réactions de fusion. Pour cela, la « bille de D$\mathrm{T}$ » est placée au centre d'une cavité cylindrique centimétrique, généralement en or (choisi pour son rendement de conversion X élevé). L'ensemble cavité + microballon est disposée au centre d'une chambre d'expérience qui mesure près de $10 \mathrm{~m}$ de diamètre (voir Fig. 1).

Cette chambre supporte les 60 quadruplets formant les deux cent quarante faisceaux lasers du Laser Mégajoule de $7.5 \mathrm{~kJ}$ chacun soit un total de 1.8 MJ. Ces faisceaux sont focalisés au travers de deux trous opposés de la cavité cylindrique et répartis pour éclairer de façon uniforme ces parois internes (voir Fig. ${ }^{\circ} 2$ ) [1]. La durée d'impulsion est de 20 ns soit, compte tenu de l'énergie délivrée, une puissance de 0.9 TW injectée dans la cavité en Or. Sous l'effet du rayonnement laser du LMJ, la cavité va produire un plasma d'or qui va restituer une partie de l'énergie laser déposée sous forme de rayons X. La cible de D-T est alors implosée par le rayonnement $\mathrm{X}$ thermalisé par la cavité à la manière d'un four.

Le plasma de D-T produit par les rayons X sur la cible se détend alors violemment et une onde de choc convergente (implosion) se propage vers le cœur de la cible comprimant le mélange gazeux devenu plasma jusqu'à atteindre les conditions de densité et de température suffisantes pour produire des réactions de fusion.

Afin analyser les divers mécanismes physiques mis en œuvre lors de ces expériences, des diagnostics plasmas [2] seront introduits dans la chambre LMJ autour de la cible à observer à l'aide de Systèmes d'Insertion de Diagnostics (SID). Ces systèmes, implantés dans le plan équatorial et dans la partie supérieure de la chambre d'expérience, permettent d'intervenir sur les diagnostics et également de les positionner précisément par rapport à la cible (Fig. $\left.\mathrm{n}^{\circ} 1\right)$.

En attendant que les installations du LMJ soient opérationnelles (2010) le CEA dispose d'une installation prototype nommée la Ligne d'Intégration Laser (LIL). Elle est constituée d'une ligne laser identique à celles qui prendront place sur le LMJ, de système d'insertion de diagnostic et d'une chambre 


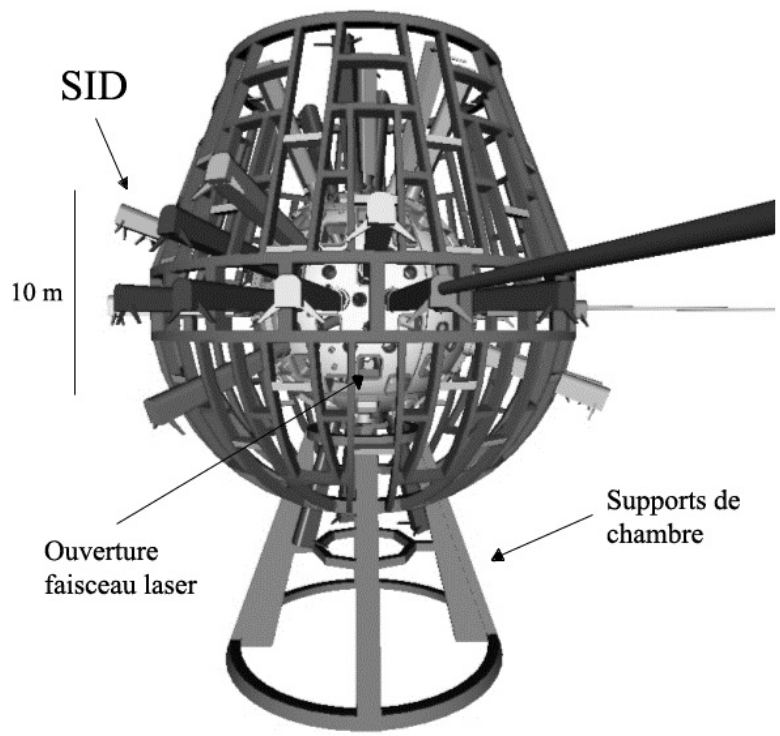

Figure 1. Maquette de la chambre d'expérience.

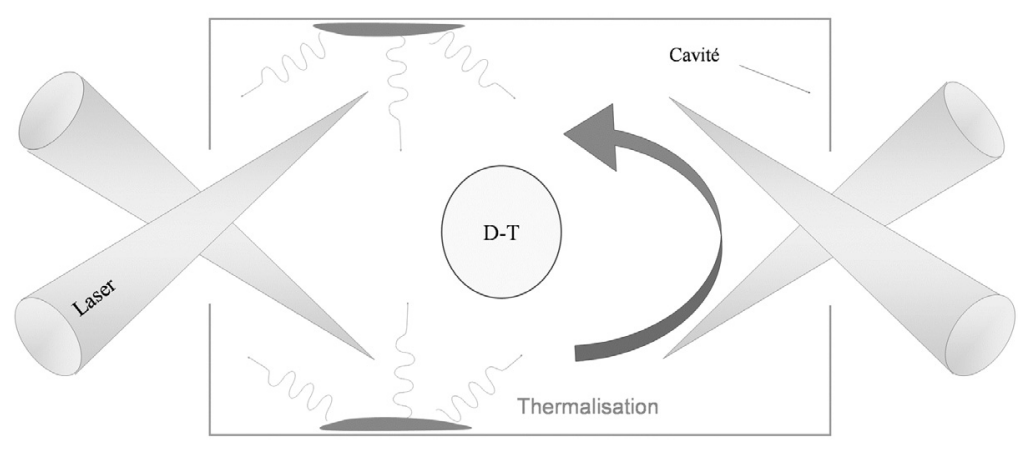

Figure 2. Principe d'une cavité - FCI par attaque indirecte.

d'expérience (de dimensions réduites par rapport au LMJ). Cette installation permet de mener diverses expériences d'interaction laser matière, de développer technologies pour l'installation LMJ et également de développer et qualifier plusieurs diagnostics plasmas dans des conditions proches de celle qui seront rencontrées sur le LMJ. Parmi eux se trouvent les Systèmes d'Imagerie X (SIX) qui font l'objet de cet article.

\section{LES SYSTÈMES D'IMAGERIE X (SIX)}

\subsection{Caractéristiques des SIX}

Un SIX comprend en général trois éléments : un système de formation d'image (par ex : sténopé, ouverture codée, optique à miroirs), un système assurant la sélection spectrale du signal (ex : filtre, 


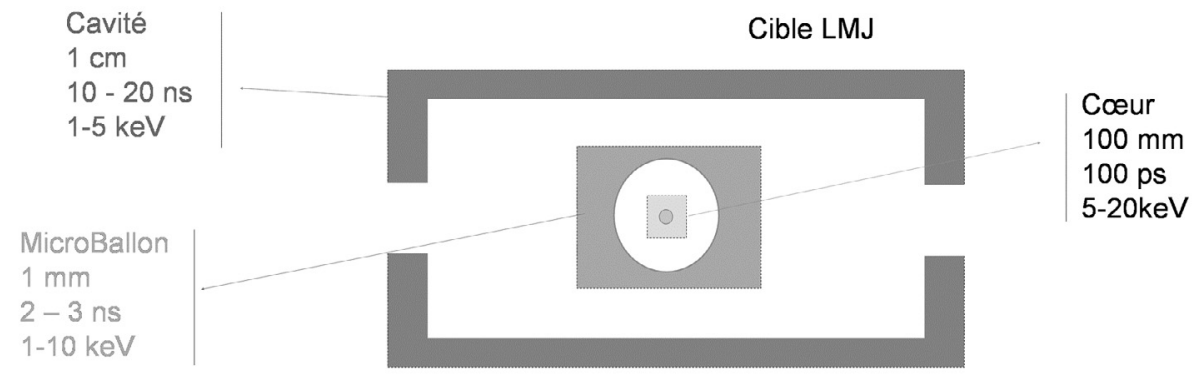

Figure 3. Schéma des zones de la cible LMJ en attaque indirecte.

miroir multicouche, réseau, cristal) et un capteur (ex : films, caméra à balayage de fente (CBF), caméra à images intégrales (CII)).

Les caractéristiques physiques des SIX sont intimement liées aux échelles spatiales et temporelles à observer lors des expériences FCI et donc aux dimensions caractéristiques de la cible. Celles-ci sont présentées dans la figure 3.

Ainsi, nous pouvons dégager trois domaines de résolution selon le champ de la cible à analyser:

- Pour la cavité, les dimensions sont centimétriques (résolution demandée moins importantes > $50 \mu \mathrm{m}$ ) et les temps d'observation de la vingtaine de nanosecondes ;

- pour le microballon les dimensions sont millimétriques (résolution demandée 10-30 $\mu \mathrm{m}$ ) et les durées de mise en vitesse sont d'environ 2 nanosecondes ;

- pour le cœur comprimé, très chaud $(5-20 \mathrm{keV})$, les diagnostics doivent mesurer avec une très haute résolution ( 2 à $5 \mu \mathrm{m}$ ) des phénomènes se produisant sur des dimensions de l'ordre de $100 \mu \mathrm{m}$ et des durées de l'ordre de la centaine de picosecondes.

L'observation de ces domaines peut être réalisée : soit par l'imagerie X liée à l'émission propre des plasmas produits (suivie de traceurs); soit par des techniques de radiographie $\mathrm{X}$. Cette dernière consiste à utiliser une source auxiliaire intense de rayonnement $\mathrm{X}$ pour sonder l'état du plasma. Cette source doit par ailleurs émettre dans un domaine spectral où le milieu à étudier est relativement transparent soit, dans le cadre des applications LMJ, entre 5 et $20 \mathrm{keV}$.

Tableau 1. Géométrie et spécification des SIX par miroirs.

\begin{tabular}{lcccc}
\hline \hline Optique & $\begin{array}{c}\text { Géométrie } \\
\mathrm{a}(\mathrm{cm}), \mathrm{b}(\mathrm{m}), \\
\gamma, \theta\left(^{\circ}\right)\end{array}$ & $\begin{array}{c}\text { Energie } \\
\text { Photons }(\mathrm{keV})\end{array}$ & $\begin{array}{c}\text { Résolution }(\mu \mathrm{m}) / \\
\text { Champ }(\mathrm{mm})\end{array}$ & $\begin{array}{c}\text { Ouverture } \\
(\mathbf{m r a d})\end{array}$ \\
\hline Sténopé LIL & $8,0.4,5$ & $\geq 1$ & $20 / 10$ & 0.25 \\
KBA Silice & $25,2.5,10,2^{\circ}$ & $\leq 0.86$ & $7 / 2$ & 2 \\
KBA Multicouche & $23,3.3,12,2.16$ & $3.1-4$ & $7 / 2$ & 2 \\
Pseudo-Wolter & $24,4,16,0.6$ & $0.1-6$ & $3 / 0.5$ & 0.8 \\
Sphère-Tore 1.05 & $72,4.13,5.7,0.85$ & $1-5$ & $30 / 3$ & 0.5 \\
Sphère-Tore 1.08 & $75,4.13,5.5,1.8$ & $0.1-1$ & $30 / 3$ & 1 \\
MKB 1.06 & $40,1.6,8,0.8$ & $1-5$ & $10 / 1$ & 0.5 \\
MKB 1.09 & $40,3.2,16,1.8$ & $0.1-1$ & $10 / 1$ & 0.5 \\
PIXEL (LIL) & $83,7.85,9,0.6$ & $0.1-10$ & $5 / 0.5$ & 0.8 \\
PIXEL (LMJ) & $79,13.4,15,0.6$ & $0.1-10$ & $5 / 0.5$ & 0.8 \\
\hline \hline
\end{tabular}

Le tableau $n^{\circ} 1$ résume la liste des SIX développés au CEA ainsi que leurs principales caractéristiques. Dans ce tableau, les valeurs expérimentales de résolution et de champ correspondent 


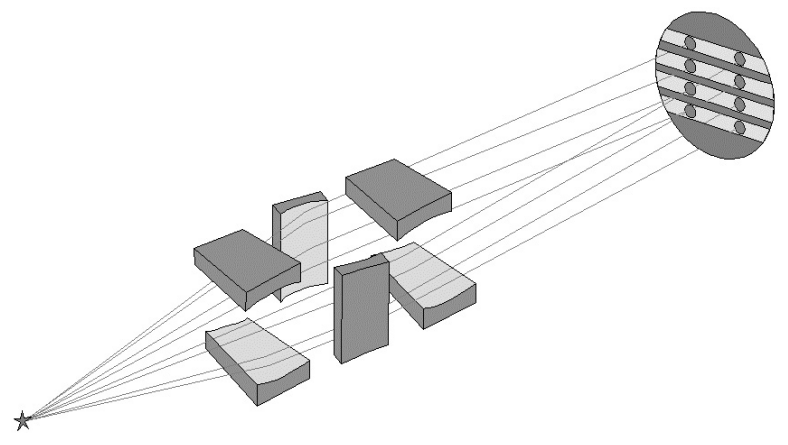

Figure 4. Imagerie par un microscope de type multi KB sur caméra à images intégrales.

à un contraste de plus de 50\%. Les valeurs nommées a, b représente respectivement la distance objet - microscope et image - microscope, $\gamma$ correspond au grandissement du système optique et $\theta$ l'angle d'attaque. Cette synthèse permet de comparer les microscopes à miroirs sous incidence rasante au sténopé utilisé dans les SIX. L'on constate sur le tableau que les sténopés, bien qu'étant simple d'utilisation, sont des systèmes peu lumineux à cause de leurs faibles ouvertures, et, si elles permettent d'imager des champs objets relativement étendus de l'ordre $\mathrm{du} \mathrm{cm}$, elles n'atteignent pas des résolutions suffisantes $(20 \mu \mathrm{m})$ pour couvrir l'ensemble des problématiques liées à la FCI.

Ainsi, la force des systèmes à miroir sous incidence rasante réside dans leur pouvoir résolvant et leur luminosité qui reste bien supérieur aux sténopés. Enfin, ils permettent un éloignement relatif du système optique de la source d'émission tout en conservant résolution et champ. Cette dernière propriété est importante en terme de vulnérabilité des diagnostics pour les futures expériences FCI.

Notons que l'étude de la physique du cœur des cibles est la plus contraignante pour les SIX par la faible dimension et par la faible durée des émissions $X(<100 \mathrm{ps})$. C'est ce domaine qui nécessite aujourd'hui des études amont : ceci sera illustré par l'exemple du diagnostic PIXEL en cours de réalisation.

\subsection{Les diagnostics de la LIL}

Le premier des systèmes à miroir développé en 1948 par P. Kirkpatrick et A.V. Baez pour l'imagerie X, comporte deux miroirs sphériques concaves "croisés" dont les plans d'incidence sont perpendiculaires entre eux [3]. Cette disposition des miroirs permet d'éliminer l'astigmatisme produit par un miroir concave seul en incidence rasante. Cette optique est couramment appelée microscope de Kirkpatrick et Baez (en abrégé, un KB). Le KB atteint une résolution de quelques microns que dans un champ assez réduit $(100 \mu \mathrm{m})$ par suite de l'inclinaison de champ de ce type de dispositif. Deux types de diagnostics, développés pour la LIL, comportent des KB mais avec des améliorations pour augmenter le champ :

- Les diagnostics multi - imagerie X en 2D avec résolution temporelle ( ${ }^{\circ} 1.06-1.09$ ) (cf. Tab.1) comprennent plusieurs couples de miroirs de type KB et forment simultanément plusieurs images dans une direction privilégiée correspondant à l'ouverture du détecteur (Fig. 4).

Pour ces multi-imageurs, le champ a été augmenté par la réduction de l'ouverture numérique. Le détecteur, une caméra à images intégrales (ou tube obturateur), permet de sélectionner temporellement une séquence d'images.

- Pour les diagnostics mono-imageurs en 1D (DP 1.05 et 1.08), nous avons souhaité augmenter le champ objet et la résolution dans une dimension $(3 \mathrm{~mm})$ tout en conservant l'ouverture. Une 


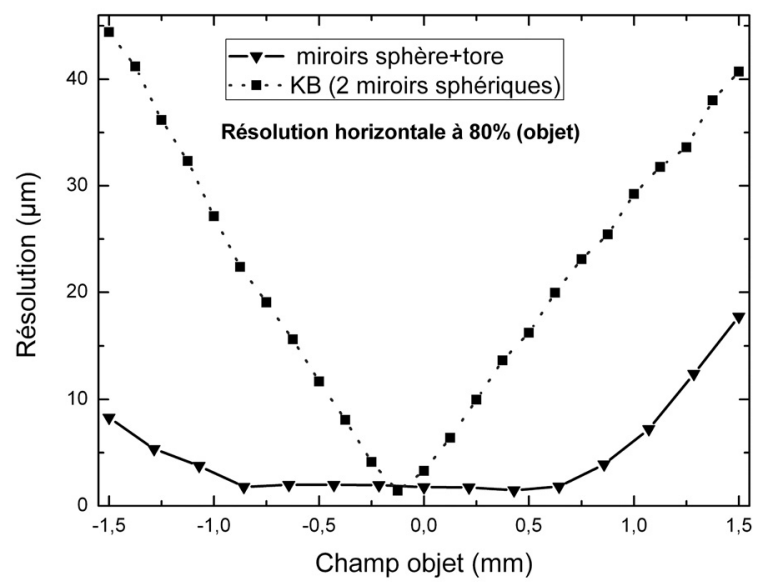

Figure 5. Évolution comparée de la résolution horizontale théorique du microscope sphère-tore (triangle), et de celle d'un KB (carré).

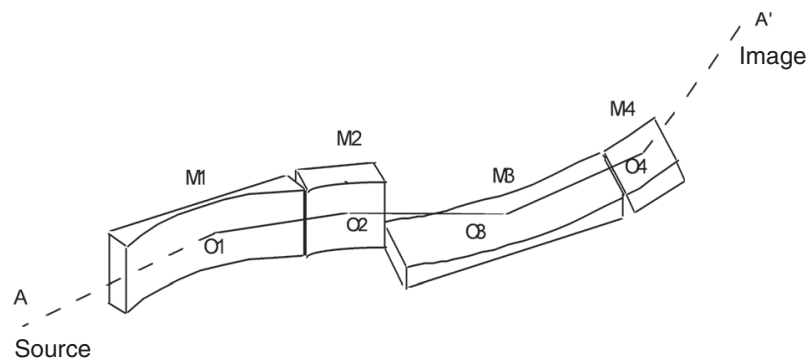

Figure 6. Schéma de principe du microscope KBA.

nouvelle formule optique a donc été déterminée grâce à des codes de logiciel de tracé de rayons adaptés aux rayons X (TRACE [4]). Elle consiste en un couple de miroir sphère-tore qui améliore considérablement la résolution dans une dimension par rapport au KB classique comme l'illustre la figure $\mathrm{N}^{\circ} 5$.

\subsection{Le microscope de type KBA}

Dans la même optique, une autre possibilité pour s'affranchir des défauts d'image et de la faible ouverture des systèmes de type $\mathrm{KB}$, consiste à utiliser non plus 2 mais 4 miroirs croisés 2 à 2 conformément à la Figure 6 ; Ce système optique appelé un KBA (pour KB Amélioré) a été réalisée par l'IOTA* en 1992 [5].

Ce microscope a des propriétés optiques très intéressantes pour l'imagerie $\mathrm{X}$ en $2 \mathrm{D}$ de par son grand champ et sa très haute résolution (de l'ordre de $7 \mu \mathrm{m}$ dans un champ de $2 \mathrm{~mm}$ ). Il présente, comme le montre le tableau 1 l'ouverture la plus élevée des différents systèmes.

Dans la perspective LMJ, l'IOTA a réalisé un second KBA attaqué sous un angle de $2^{\circ}$. Pour des raisons de luminosité du système à 4 miroirs, il a été nécessaire d'atteindre un pouvoir réflecteur pour

* Institut d'Optique Théorique et Appliqué, 91405 Orsay Cedex, France 
chaque miroir de près de $10 \%$ [6]. Un tel pouvoir réflecteur ne peut être obtenu avec de simples couches métalliques: on obtient $0,4 \%$ à $3,4 \mathrm{keV}$ pour une couche de tungstène à $2^{\circ}$. Les 4 miroirs ont donc été revêtus de multicouches pour sélectionner une bande passante de $1 \mathrm{keV}$ autour de $3,4 \mathrm{keV}$. Cette bande passante permet de travailler par radiographie sur la raie d'émission d'un plasma d' Ag (3, 4 keV) ou le suivi de traceurs d'Argon (3, $7 \mathrm{keV})$.

Une difficulté de réalisation de ce microscope a été d'obtenir un éclairement constant dans le champ. Dans le cas des miroirs en silice (premier KBA), le pouvoir réflecteur varie peu en fonction de l'incidence, ce qui n'est pas le cas avec un traitement multicouches périodique. L'alternative possible a été de réaliser un empilement d'épaisseurs non périodiques calculées de manière à générer un pouvoir réflecteur le plus constant possible dans le domaine angulaire et spectral voulu. Ce microscope est en cours de test sur un de nos bancs d'imagerie et présente une résolution spatiale importante au centre du champ proche de $3 \mu \mathrm{m}$ !

Pour certaines applications, la faible luminosité des KBA peut s'avérer être un inconvénient. Le microscope de Wolter constitué de deux miroirs asphériques (elliptiques) est la solution alternative théoriquement la plus performante [7]. Malheureusement il est très difficile à réaliser car les tolérances de fabrication associés sont très sévères, tant pour la forme des asphériques que pour l'état de surface. $\mathrm{La} \mathrm{SESO}^{\dagger}$ a réalisé pour le CEA un Pseudo-Wolter [8] associant deux portions de tores à symétrie de révolution qui permettent de s'approcher au mieux des performances du Wolter classique. Ceci a nécessité de réduire l'ouverture du faisceau par une pupille d'entrée (voir Tableau 1). Ce microscope, aujourd'hui opérationnel dans le diagnostic High Resolution X-rays Imaging (HRXI) sur la chambre d'expérience du laser Omega du LLE ${ }^{\ddagger}$, est un bon compromis résolution-efficacité. Ce principe de microscope a été retenu pour la conception du bloc optique du diagnostic PIXEL.

\subsection{Le diagnostic PIXEL}

\subsubsection{La formule optique}

Le diagnostic PIXEL aura pour fonction de faire l'imagerie 1D (résolue en temps) et 2D du plasma dense des cœurs de cible LMJ en phase de combustion.

Compte tenu du fait que l'énergie libérée sera beaucoup plus importante sur le LMJ que sur les lasers actuels, la distance minimum d'approche des microscopes sera plus grande afin d'éviter leur détérioration pendant les tirs. En effet, les expériences de fusion conduiront à l'émissions de neutrons $(14 \mathrm{MeV})$ de gamma (3.5 MeV) et débris du plasma d'or, ce qui limitera le tirage mécanique objet du microscope à $800 \mathrm{~mm}$. Pour les mêmes raisons, les détecteurs doivent être éloignés au maximum de la source émissive. Le plan image sera donc situé à l'arrière des systèmes d'insertion SID (voir Figure $\mathrm{n}^{\circ} 7$ ) soit environ $13 \mathrm{~m}$ de la cible et donc le grandissement maximum du microscope sera d'environ $\sim 15 x$.

Le champ à observer, défini par le cœur de la cible en phase d'implosion, est de l'ordre de $500 \mu \mathrm{m}$ de diamètre. La résolution attendue est de $5 \mu \mathrm{m}$. Cette résolution est compatible avec la résolution du détecteur compte tenu du grandissement du microscope. La gamme spectrale de fonctionnement choisie pour PIXEL est de 5 à $10 \mathrm{keV}$. L'angle d'attaque de $0.6^{\circ}$ choisi pour les miroirs, est un compromis entre la nécessité de diminuer l'angle d'attaque pour réfléchir les hautes énergies et la nécessité de limiter la dimension longitudinale des miroirs.

L'étude et la réalisation opto-mécanique du microscope ont été confiées à la société Winlight Système $^{\S}$ (WLS). Contrairement au Pseudo-Wolter, il a été nécessaire d'avoir trois miroirs toriques M1, M2 et M3 attaqués à $0,6^{\circ}$ conformément à la figure $n^{\circ} 8$, pour conserver les spécifications de $5 \mu \mathrm{m}$ de résolution spatiale et pour avoir des rayons de courbure réalisables.

\footnotetext{
** Société Européenne de Systèmes Optique, Aix-en-Provence, France

*** LNE, ...

**** Société Winlight System S.A., Village d'Entreprises Saint Henri, Bât. 301-302, 13016 Marseille Cedex, France
} 


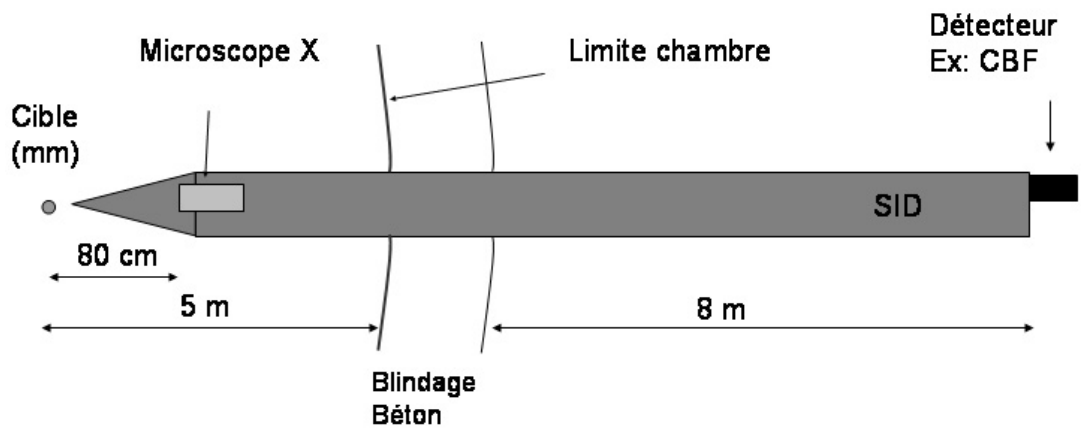

Figure 7. Schéma d'implantation du diagnostic PIXEL.

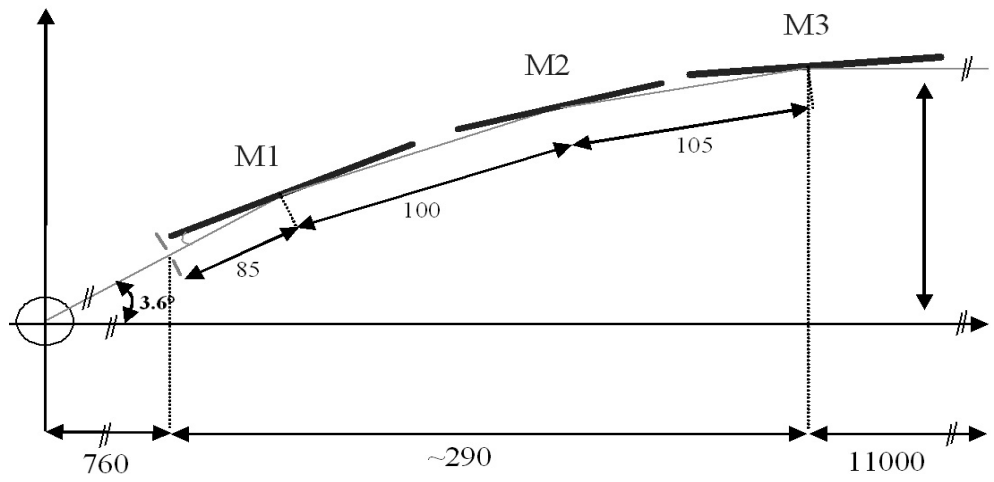

Figure 8. Schéma de la formule optique du microscope du diagnostic PIXEL.

Ce microscope est un système hors axe, orienté avec un angle de $\beta=3.6^{\circ}$ par rapport à l'axe du Système d'Insertion de Diagnostique SID. Cet angle est nécessaire pour faire ressortir le faisceau parallèle à l'axe de l'inserteur. Les caractéristiques optiques des miroirs sont données dans le Tableau $\mathrm{n}^{\circ} 2$.

Tableau 2. Caractéristiques des miroirs du microscope X du diagnostic PIXEL.

\begin{tabular}{llcr}
\hline \hline Miroirs & Type & Rt $(\mathrm{m})$ & Rs $(\mathrm{mm})$ \\
\hline Ma151 -1 & Torique biconcave & 574 & 62 \\
Ma151 -2 & Torique biconcave & 348 & 36 \\
Ma151 -3 & Torique biconcave & 639 & 78 \\
\hline \hline
\end{tabular}

Notons dans ce tableau les dimensions importantes des rayons de courbure tangentiels qui sont à la limite des technologies actuelles : de contrôle des optiques et de polissage.

La figure 9 présente la réponse du microscope à une source ponctuelle pour le grandissement 9 . L'image est observée dans le plan focal image du microscope (8749.3 mm de M3) au niveau du cercle de moindre diffusion (C.M.D.) et dans les deux plans focaux sagittal et tangentiel (Fig. 9).

Les dimensions des taches au niveau du CMD de ces trois plans sont récapitulées dans le tableau 3.

Les simulations montrent que cette formule optique permet d'obtenir des résolutions (objet) (hors diffraction) inférieures au $\mu \mathrm{m}$, ceci dans tout le champ objet de $500 \mu \mathrm{m}$ de côté. Elle possède deux 


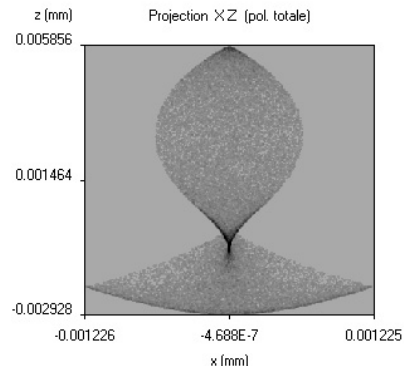

Foc. Sag.

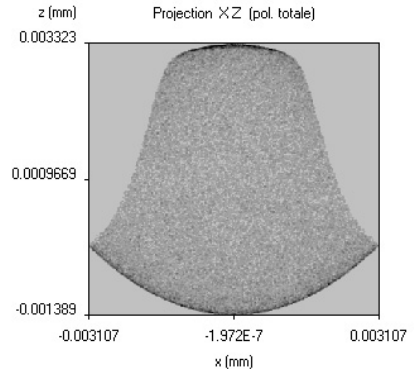

C.M.D.

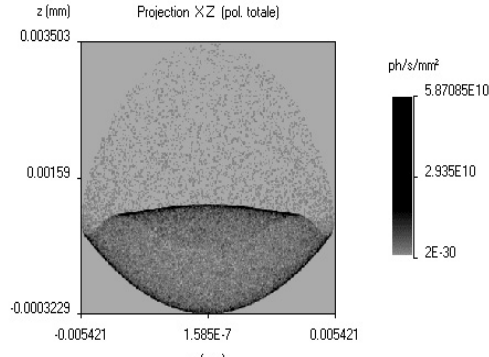

Foc. Tg.

Figure 9. Spots diagrams.

avantages : une grande profondeur de champ (centimétrique coté objet), et une plage de grandissement allant de 9 à 15. Elle présente un très léger astigmatisme au fort grandissement et une aberration géométrique de type coma qui dégrade peu l'image en bord de champ.

L'analyse de l'image simulée d'une grille par la méthode dite du bord franc indique une résolution optique (hors diffraction) inférieure au $1.8 \mu \mathrm{m}$ dans tout le champ objet de $1 \mathrm{~mm}$ de côté.

Tableau 3. Dimension des taches focales et résolution théorique au niveau des plans focaux.

\begin{tabular}{lcc}
\hline \hline Type & $\mathbf{X ~ ( 8 0 \% )} \boldsymbol{\mu \mathbf { m }}$ & $\mathbf{Z}(\mathbf{8 0 \%}) \boldsymbol{\mu m}$ \\
\hline Tache - Plan Focale Sagittale (x) & 1.16 & 8.4 \\
Tache - Plan Focale Tangentielle (z) & 8.32 & 2.16 \\
Tache - C.M.D & 4.08 & 5.01 \\
Résolution éq. Sagittale (x) & 0.077 & 0.564 \\
Résolution éq. Tangentielle (z) & 0.554 & 0.144 \\
Résolution éq. CMD & 0.272 & 0.334 \\
\hline \hline
\end{tabular}

\subsubsection{Traitement multicouche des miroirs de PIXEL}

L'angle d'attaque de $0.6^{\circ}$ ne permet pas la réflexion totale des énergies supérieure à $5 \mathrm{keV}$. Il est donc nécessaire d'utiliser des revêtements multicouches adéquats $[9,10]$ pour accroître le domaine spectral de réflectivité des miroirs. Il s'agit de structures interférentielles apériodiques appelées Super Miroirs [11, 12].

De nombreuses simulations et optimisations, basées sur un formalisme matriciel développé par F. Abèles en 1967 [9-12] ont été réalisées sur plusieurs empilements multicouches différents. Le revêtement choisi est un empilement apériodique de Tungstène/Silicium W/Si. Le but étant d'obtenir un super miroir dont le pouvoir réflecteur excède 30\% sur la plus large bande spectrale au dessus de $5 \mathrm{keV}$. La formule multicouche a été testée au centre de rayonnement synchrotron de BESSY [13]. Le pouvoir réflecteur en fonction de l'énergie des photons incidents est donné dans la figure $n^{\circ} 10$.

La courbe expérimentale présentée est comparable aux courbes de réflectivités calculées. La formule de l'empilement multicouche permet d'obtenir une réflectivité très uniforme, supérieure à $40 \%$ entre 5 et $9.5 \mathrm{keV}$, et proche de $30 \%$ entre 9.5 et $10 \mathrm{keV}$. Notons que la coupure du miroir aux alentours de $10.2 \mathrm{keV}$ est liée au seuil d'absorption du tungstène en couche L. Le revêtement a été réalisé en collaboration avec le Laboratoire de Microélectronique et de Matériaux de Provence (L2MP).

\subsubsection{Mécanique du bloc optique du microscope du diagnostic PIXEL}

Le bloc optique du diagnostic PIXEL est équipé d'une mécanique de haute précision permettant en plus des mouvements propres du SID son positionnement spatial dans la chambre d'expérience. 


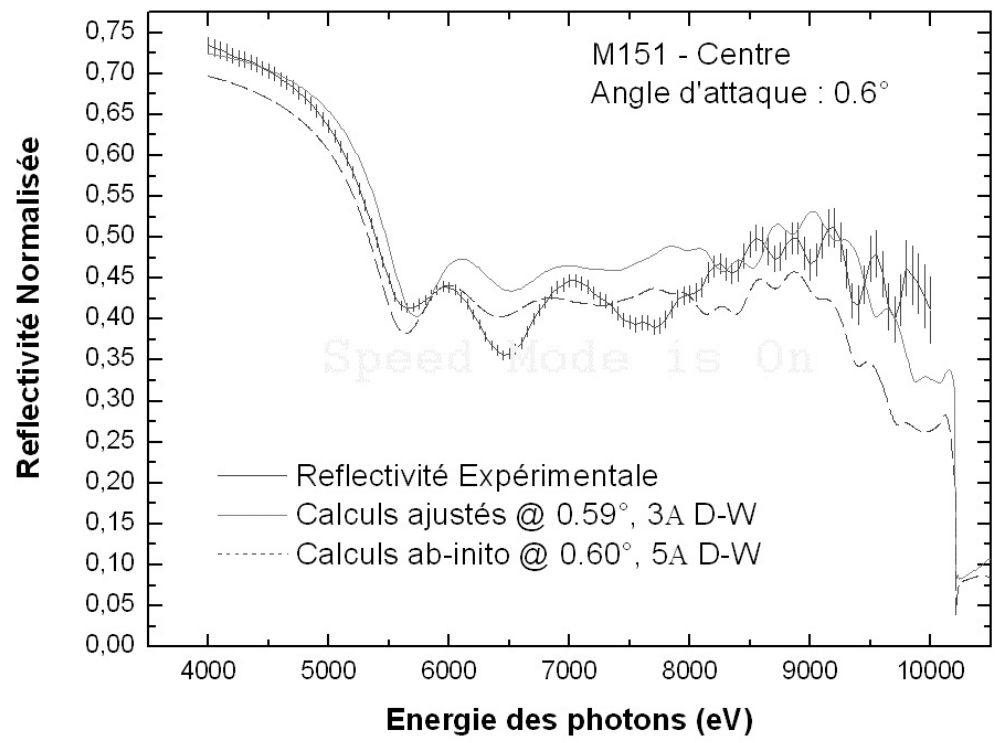

Figure 10. Pouvoir réflecteur (trait plein) comparé aux calculs ab-initio (trait tiré) et ajusté (trait pointillé).

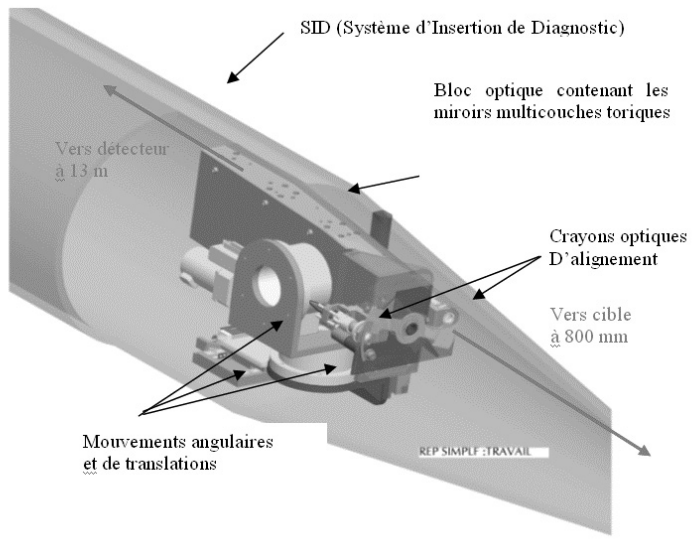

Figure 11. Schéma d'implantation du microscope et de sa mécanique dans un SID.

Il est également équipé d'un système d'alignement intégré destiné à faire coincider le foyer objet du microscope avec la cible. Ce système comporte des crayons optiques (diode laser) placés de part et d'autre de la pupille d'entrée réglée en laboratoire pour converger vers le foyer du microscope. Ils permettent le réglage en pointage et en tirage du microscope sur la cible avec des précisions d'alignement de l'ordre de 50 à $100 \mu \mathrm{m}$ soit approximativement $1 / 10$ du champ objet. Un crayon optique situé à l'arrière du microscope indique la position de l'image permettant l'alignement des détecteurs (caméra).

Le diagnostic PIXEL pourra être utilisé en imagerie 2D et 1D résolue en temps. Pour l'imagerie $2 \mathrm{D}$, une caméra CDD X à haute résolution sera utilisée $(13 \mu \mathrm{m}: 2$ pixels de $7 \mu \mathrm{m})$. Pour l'imagerie $1 \mathrm{D}$ résolue en temps (cinématique ultrarapide), des caméras à balayage de fente développées au CEA seront utilisées. 


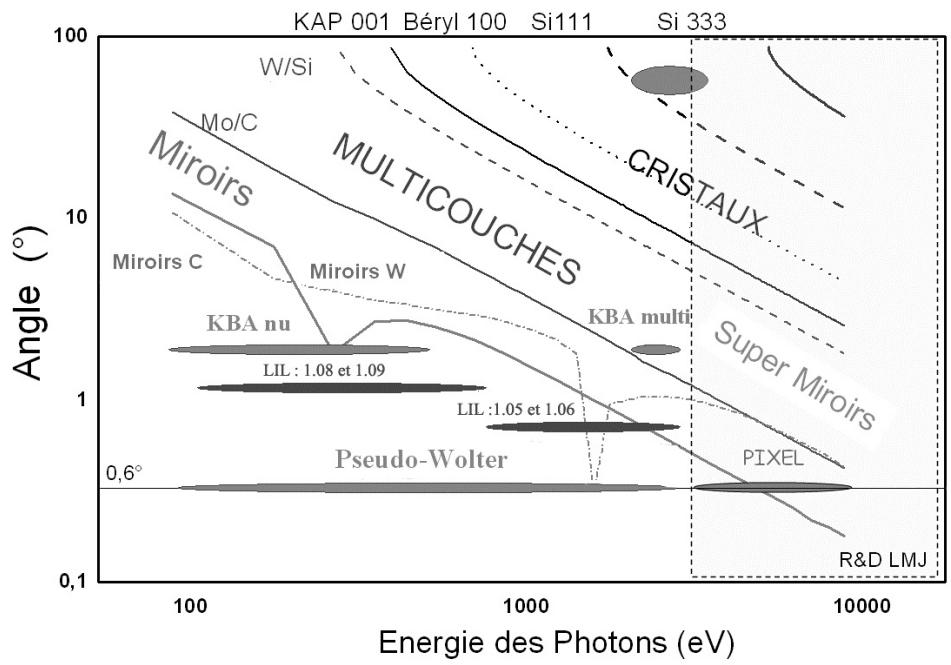

Figure 12. Diagramme de Dumond des optiques utilisées dans les SIX.

\section{CONCLUSION}

Plusieurs diagnostics d'imagerie ont été développés au CEA pour l'étude des plasmas produits par laser. Les principaux systèmes fabriqués ou en projet ainsi que leurs caractéristiques angulaires et énergétiques sont répertoriés dans le diagramme de Dumond Fig. 12. Pour les miroirs, l'angle d'attaque doit être compris entre une limite basse $\left(0,5^{\circ}\right)$ liée aux difficultés d'alignement, à l'augmentation des aberrations géométriques et aux dimensions des optiques, et une limite haute de quelque degrés lié à l'absorption des rayons $\mathrm{X}$. On voit aussi que le domaine de prédilection de $5-20 \mathrm{keV}$ impose de travailler à des angles d'attaque très rasants proche de la limite basse. Les optiques de type super-miroirs ou cristaux seront donc privilégiées pour la conception des diagnostics imageurs du LMJ. Les systèmes réfractifs (Lentilles à bulles) et diffractifs (Lentilles de Fresnel) seront également explorés.

D'une manière générale, les challenges technologiques seront de réaliser des diagnostics robustes capables de résister aux contraintes d'environnement liées aux 20 MJ dégagés par une cible à gain.

\section{Remerciements}

Ces travaux ont été réalisées grâce à de nombreuses collaborations du CNRS : l'IOTA (F. Bridou et R. Mercier), le L2MP (B. Vidal, T), avec des industriels SESO et Winlight System.

\section{Références}

[1] Chocs 2004, « Le laser mégajoule, un élément clef du programme simulation », Revue Scientifique et Technique des applications militaires, 29-2

[2] P. Troussel, "Quelques diagnostics pour les expériences d'interaction laser-matière", Annales de Physique $27 \mathrm{~N}^{\circ} 3$ (2002)

[3] Kirkpatrick P., Baez A.V., « Formation of Optical Images by X-Rays », J.O.S.A. 38 (9) (1948) 766-774

[4] TRACE : T. Moreno (société CAMINOTEC) 2001.

[5] Sauneuf R. "Large field high resolution X-ray microscope for studying laser plasmas", Rev. Sci. Instrum. 68 (9) (1997) 3412 
[6] F. Bridou et al "Large field double Kirpatrick-Baez microscope with nonperiodic multilayers for laser plasma imaging” Rev. Sci. Instrum. 73 (11) (2002)

[7] H. Wolter, Ann. Der Phys. 10 (1952) 94 et 256

[8] Ph. Troussel et al, "Wolter-like resolution x-ray imaging microscope for Rayleigh Taylor instabilities studies" Rev. Sci. Instrum, 76 (2005) 063707

[9] E. Spiller, Appl. Phys. Lett. 20 (1972) 365

[10] A. V. Vinogradov et al., Appl. Opt., 16 (1977) 89

[11] K.D. Joensen \& al., Nucl. Instr. Meth. B 132 (1997) 221

[12] P. Hoghoj \& al., Nucl. Instr. Meth. B 132 (1997) 528

[13] J. Ph. Champeaux \& al, « Development and realization of non-periodic W/Si multilayer for 5-14 keV X-rays plasma diagnostic », J. Opt. Commun 27 (2006) 001-005

[14] Vidal B. et al., "Development of multilayer optics at L2MP", Communication at COST P7 meeting, 2005 